\title{
Proteolytic Transformation and Stimulation of SARS- Cov-2 Spike Protein with Human ACE-2 Receptor
}

\author{
Sohail S'*, Rana $\mathrm{H}^{2}$, Awan DS ${ }^{3}$, Sohail F, Rishi \\ $\mathbf{A I}^{3}$, Karamat $\mathrm{M}^{5}$, Khan $\mathrm{S}^{6}$ and Adil $\mathrm{K}^{7}$ \\ ${ }^{1}$ MS, Microbiology, University of Central Punjab, Pakistan \\ ${ }_{2}^{2}$ MBBS, Allama Iqbal Medical College, Pakistan \\ ${ }^{3}$ MBBS, Rawalpindi Medical University, Pakistan \\ ${ }^{4} \mathrm{BS}$, Microbiology, Government College University, \\ Pakistan \\ ${ }^{5}$ MBBS, King Edward Medical University, Pakistan \\ ${ }^{6}$ Biotechnology, University of Central Punjab, Pakistan \\ ${ }^{7} \mathrm{BS}$, Applied Microbiology, University of Veterinary and \\ Animal Sciences, Pakistan
}

*Corresponding author: Shehreen Sohail, MS Microbiology, University of Central Punjab, Pakistan

Received: September 24, 2021; Accepted: October 29, 2021; Published: November 05, 2021

\begin{abstract}
Severe acute respiratory syndrome coronavirus has a great role in causing respiratory illness in humans and has the most important relationship of its spike proteins with host ACE-2 receptors. After entry into the human body, the viral $S$ protein receptor-binding domain binds to human ACE-2 receptor. Two modes explained in this paper of an ACE- 2 shedding. The shedding induces the process of viral entry to host cells by binding SARS-CoV-2 proteins. The residues of arginine and lysine in the ACE-2 receptor from 652 to 659 amino acid cleavage by ADAM17 but in TMPRSS2 the residues can be seen on amino acid from 697 to 716 . Corona virus genome shows some structural proteins that are responsible for the cellular entry and facilitate the attachment of a virus to the host cell. Virus recognizes the attachment site and binds with it and enter into the cell. Spike protein is split from the cleavage site along its two subunits $\mathrm{S} 1$ and S2 then during this process. S2 subunit release RBD (Receptor- Binding Domain) of S1 mediated to the ACE-2. The RBD of S1 consists of 200 amino acid domains. The unknown protein $\mathrm{B}^{6} \mathrm{ATI}$ which is a neutral amino acid transporter located in ileum is the basic cause for formation of ACE-2 homodimer. In this way S1 domain provides site for another S2 domain. This leads to concealing of the ACE-2 ectodomain cleavage-sites, shedding. It prevents endocytosis of the receptor blocking a major pathway in the viral entry.
\end{abstract}

Keywords: Spike proteins; Receptor binding domain; Angiotensinconverting enzyme 2; Viral entry; Corona virus; Protein

\section{Introduction}

On $1^{\text {st }}$ December 2019, the patient index became symptomatic for the deadly Novel Coronavirus in the Wuhan city of China, called off as Chicago of China. Coronavirus is one of the 124 viruses in Coronaviridae family. It is an enveloped RNA virus. RNA is positive sense. Its glycoprotein envelope has spikes emerging out of it. This is what gives coronavirus its unique crown shape leading it to be called as corona. Earlier on, coronaviruses were regarded as causative agents of respiratory and intestinal diseases among birds and domestic animals. Now we have seen that 6 out of 124 types of Coronaviridae family were accused of causing major and minor diseases in humans. Among these 6, 4 types are associated with common cold and flu. While the other two types except these four were seen to be causing severe acute respiratory syndrome often regarded as SARS and Middle East Respiratory Syndrome (MERS-CoV). With a length ranging from 26 to $13632 \mathrm{~KB}$, coronavirus is declared to have the longest and broadest genetic material in the recent publications [1]. A large portion of its genome also leads to transcription and translation of important polypeptides for gene expression and multiplication, in addition to its major structural proteins. Numerous experiments and researches have been conducted in the recent past to achieve a good understanding of viral replication and virulence at molecular level along with its adaptations, so that it helps in development of vaccines and medicines for diagnostic and therapeutic purposes and evaluation procedures. The natural hosts of SARS-CoV-2 virus are bats. In Wuhan the food markets near sea, hospitals and clinics became the source of corona virus after the permanent residents inhaled the air droplets. There are four genera of corona virus $\alpha-/ \beta$ $/ \gamma$ - $/ \delta$-CoV. SARS-CoV- 2 is a $\beta$-coronavirus that has non-segmented and non-enveloped positive SSRNA virus. The different types of this virus affect different species like alpha and beta types infect mammals and gamma and delta affect birds. After the breakdown of this virus total six so far types are found to infect human beings. Alpha-CoV HCoV-229E and HCOV-NL 63 and Beta COV HCoV- HKU1 and $\mathrm{HCoV}-\mathrm{OC} 43$ these generally have low pathogenicity and give rise to mild respiratory symptoms resembling the common cold but the beta two types of corona virus, SARS-CoV-2 and MERS-CoV cause lethal and deadly respiratory symptoms. The genome sequencing shows $\mathrm{CoV}$ genome in bats (RaTG13) approximately $96.2 \%$ which is similar to SARS-CoV-2 79.5\%. We can assume the host for the origin of virus in humans are bats [2,3]. Angiotensin- Converting Enzyme (ACE-2) has the same receptor as the SARS-CoV-2, so it uses this enzyme while infecting humans [4]. According to genomic research SARS-CoV-2 with a genome length of 27 to $32 \mathrm{~kb}$, coronaviruses are the largest among all the RNA viruses, consisting of a non-segmented positive sense RNA. The coronavirus family, Coronaviridae consists of 4 genera; $\alpha-/ \beta-/ \gamma-/ \delta-\mathrm{CoV}$. There are 6 members of Coronaviridae family that tend to infect humans. 4 of them, $2 \alpha-\mathrm{CoV}$ (HCoV-229E and HCoV-NL63) and $2 \beta-\mathrm{CoV}$ (HCoV-HKU1 and HCoV-OC43) lead to only mild or less severe respiratory infections. Among these, the $\beta$-CoV, including SARS- CoV, MERS-CoV, and the novel SARS$\mathrm{CoV}-2$ are of prime relevance, since they infect humans. Avian species is the most infected by $\gamma-/ \delta$ - $\mathrm{CoV}[5,6]$. The virus comprising nonsegmented positive RNA is one of the largest among all RNA SARS$\mathrm{CoV}-2$ viruses. The sequenced genome of this virus is $27-32 \mathrm{~kb}$ long. 
The four genera of Coronaviridae family are differentiated by causing diseases in different species. $\alpha-\mathrm{CoV}(\mathrm{HCoV}-229 \mathrm{E}$ and $\mathrm{HCoV}-\mathrm{NL63})$ and $2 \beta-\mathrm{CoV}$ (HCoV-HKU1 and HCoV-OC43) cause mild or less severe respiratory infections. Among these, the $\beta-\mathrm{CoV}$, including SARS- CoV, MERS-CoV, and SARS-CoV-2 infect humans. Avian species is the most infected by $\gamma-/ \delta$ - $\mathrm{CoV}[5,6]$. The nucleocapsid protein is a helical capsid and lipid bilayer covering the RNA nucleocapsid core produced by M protein, E protein, and S protein [5], which are used to accommodate the Novel Coronavirus Genome. The virus gets its crown appearance because of large outwardly ectodomain of spike protein. A short intracellular tail attaches to the spike protein which is basically a single pass transmembrane. The ectodomain of $S$ protein consists of $3 \mathrm{~S} 1$ subunit heads is always responsible for receptor binding and a trimeric S2 subunit stalk usually facilitates membrane fusion, which enables the entry of virus. The spikes of coronavirus act as class I membrane fusion proteins [5]. The main core of receptor binding is always $\mathrm{S} 1$ subunit total three in number and present in the ectoderm of spike protein. Membrane fusion is always facilitated by a trimeric S2 subunit. The virus entry in host cell always happens this way. These spike proteins act as class 1 membrane fusion proteins [5] and this protein being metastable repositions its structure for bringing virus and host membranes close to each other. This all happens with a process name fusion configuration; the change and rearrangement of $S$ protein occur from pre-fusion configuration. On each side of 3 S1 subunits, the presence of RBD having different sub regions and these recognize various receptors distinctly belonging to different viral species. In order to interact with human receptors, the SB domain is used by the corona virus and many other strains associated with the family [7]. The transient exposure or down-conformation depending on $\mathrm{CoV}$ species occur through hinge like movement of SA and SBA determinants [8]. The proteases of host cell dominant the spike protein by removing two amino acids but this happens in its pre-fusion state. After binding of S1 subunit, S2 subunit detaches from S1 subunit in order to alter themselves in a post fusion stable and dumb-bell conformation [8]. The viral and host membranes are brought together by a bundle of six helices appearing from S2 subunit and attaches itself in the host. The ability of RBD corona virus helps to recognize many cell surface molecules like proteins, sugars, and heparin sulfate [9]. Carbohydrate sites are usually fixed for Nterminal of S1 subunit domain and for C- terminal there are group pf protein receptors for this purpose. The receptor for MERS-CoV is DPP4 (dipeptidyl peptidase 4; or CD26, on chromosome 2q24.2) an essential peptide hormone regulatory enzyme and $\mathrm{T}$ - cell activation antigen. Both A (SA) and B (SB) domains of the MERS-CoV S protein interact with its receptor (DPP4) [10]. There is no structural or sequential homology between ACE-2 and DPP4 [10]. Cluster of differentiation 147 is another possible receptor for SARS-CoV-2 147 [11]. Many recent studies after the outbreak of the corona virus revealed that the SARS-CoV-2 RBD and host ACE-2 receptor have always been determined and regulated by binding affinity to host virus susceptibility. Human host immune responses have an essential inducer which is mainly the S1 RBD spike $[9,12]$. The increased RBD/ human ACE-2 (hACE2) binding affinity is responsible by an amino acid substitution (K479N) in the novel corona virus (RBD) for civet to human spillover of SARS-CoV-2. Human to human transfer is most noticeable and is facilitated by mutation that is S48T in SARS-CoV-2 RBD. Many other mutations of the virus occur at five positions which are beneficial for the enchantment of S1 binding affinity with civet ACE-2 and hACE-2. In this case we can assume that viral replication rate which different in different species can link with all the binding interactions that occur between SARS-CoV S protein and hACE-2 [7].

\section{ACE-2 Receptor and Its Function}

Co immunoprecipitation studies were used to identify the role of ACE-2 receptors for corona virus after its outbreak. It a well-known procedure to recognize physiologically applicable protein-protein communications by utilizing objective protein-explicit antibodies to by implication catch proteins that are bound to a particular objective protein. In SARS-Cov-2 the RBD with more than 10-fold affinity find to the ACE- 2 receptor. ACE- 2 is basically a dipeptidyl carboxypeptidase. It is present on 805 amino acid type 1 protein and the domain with which it binds is zinc binding Domain. It is expressed in lungs of human body along with kidney, heart, testis and human gut. As corona virus is associated with respiratory tract so in a human lung ACE-2 is present in epithelial type II cells. ACE2 appears in many forms and it has another soluble form. There is no as such role of protease activity in facilitating the viral entry of SARS-Cov-2 but it more acts like a receptor to help and specify the attachment of virus and fusion of its spike protein with help of its two functional regions. The first is N-terminal M2 peptidase domain and the second one is $\mathrm{C}$-terminal collectrin domain which regulate the transfer of amino acid to cell membrane. ACE-2 perform function of conversion of angiotensin 1 to angiotensin (1-9) and also angiotensin II to angiotensin (1-7). Vasodilation and anti-inflammatory effects occur when angiotensin (1-9) binds with the Mas receptor. So, like this angiotensin II causes such effects via receptor type 1 . The co crystal structure identified and showed that interaction of spike protein RBM with human ACE-2 domain. The SARS-Cov-2 binding appeared to be more intense due to two lysine residues on ACE- 2 receptor. The creation of positive charge that is necessary to neutralized by SARSCov-2. The lysine residue virus hotspots are critical for the attachment and binding of corona virus. These residues in the RAM of SARSCoV-2, Gln493 and Leu455 bind making stabilization and binding with greater affinity for ACE-2 more than SARS-CoV-2. Another domain called ganglioside-binding domain play an important role after its identification in the $\mathrm{N}$-terminus of corona virus. This specific domain is imitated by chloroquine and hydroxychloroquine. It is also known that binding factors are important for the interaction of spike glycoprotein. In any case the increase in ACE-2 receptor expression can spread the infection in body. If there are more ACE-2 receptors in body then they can delay the binding of SARS-CoV-2 to cells and reduce injury to the cellular tissues as well.

\section{Characteristics of ACE-2 Receptors}

\section{Structure of ACE-2 receptor}

The discovery of ACE-2 in 2000 is a homolog of metalloprotease angiotensin-converting enzyme ACE [13], which was essential to act as a regulator for human heart [14]. These are transmembrane proteins having 805 chains of amino acids with presence of two domains: M2 domain having N-terminal peptidase and a C-terminal domain for collectrin renal amino acid transporter consisting zinc at active site named as HEMGH domain which has sequence homology of $48 \%$ with ACE amino domain and it is primarily involved in 

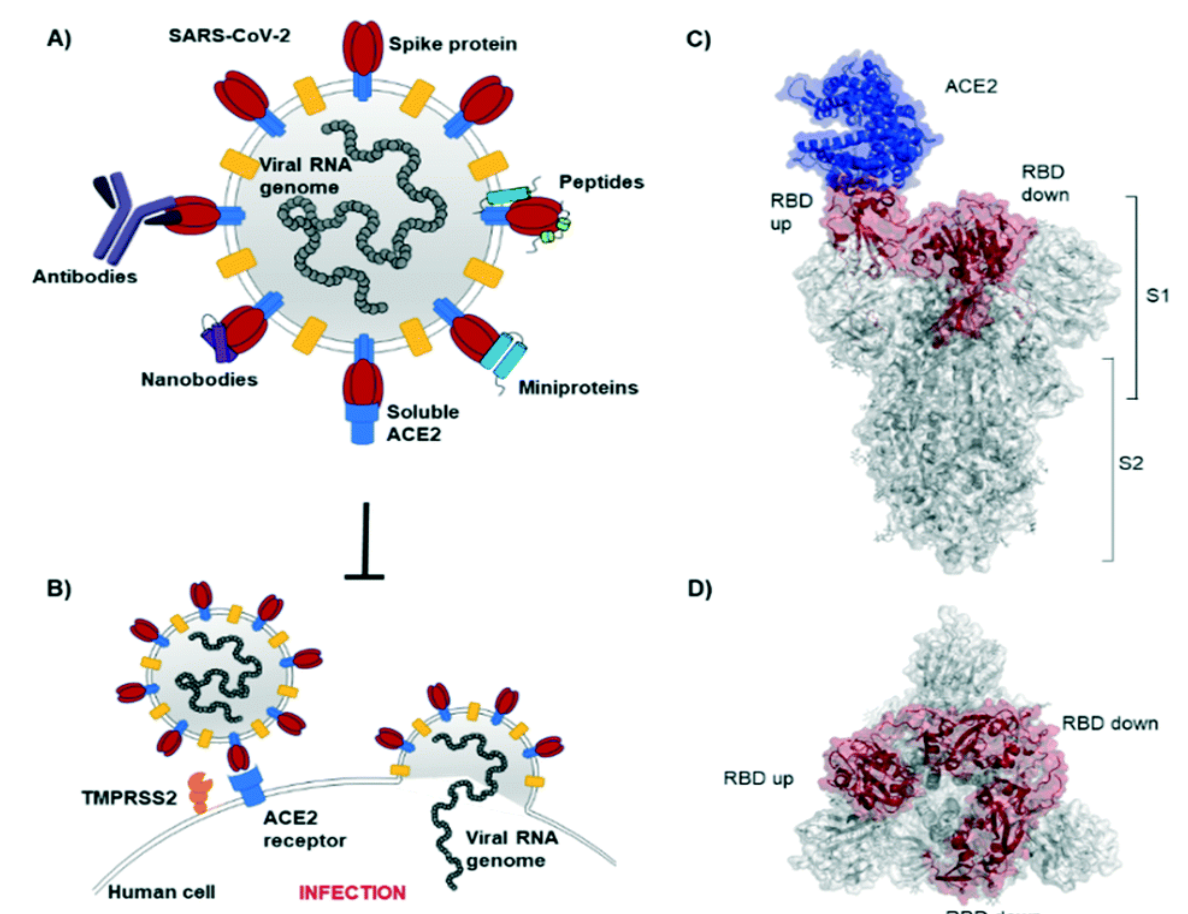

D)

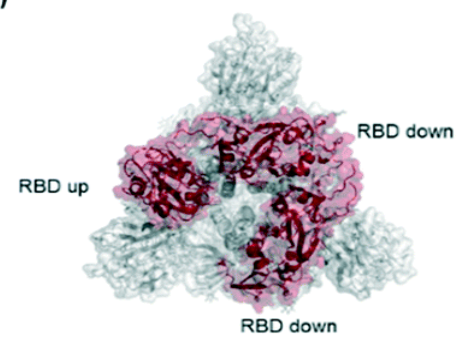

Figure 1: A SARS-CoV-2 particle bound to the spike protein is depicted schematically, In the figure, four different parts are described. In part a, there is a link between the Corona virus spike proteins binding with the ACE-2 receptor. The proteolytic cleavage which is done by TMPRSS2 results in the fusion of spike proteins with the ACE-2 receptor. The trimeric SARS-CoV-2-spike protein is shown along with ACE-2 receptors (blue) bound by one RBD (red) in an up conformation, while the other two RBDs (red) are in 'down' conformation. Figure courtesy of RSC Med. Chem., 2021, 12, 197.10.1039/d0md00385a.

formation of Angiotensin, a vasoactive peptide and corona virus $\mathrm{S}$ binding protein. The $\mathrm{C}$ - terminal domain of the receptor shows homology $48 \%$ with collectrin. Its interaction with $\mathrm{B}^{6} \mathrm{ATI}$ which is an amino acid transporter shows great importance. ACE-2 receptor has an active domain, which is present on intestines and other cells of human body. Sheddase is an enzyme which release the soluble protein in bloodstream and also in some cases it is excreted in the urine too, basically it uses the ACE-2 extracellular domain which is cleaved from the transmembrane with help of this enzyme $[15,16]$.

\section{Location of ACE2 in cells}

ACE-2 is an enzyme with its active domain to be extracellular, with scant part to be intracellular. The process of internalizing ACE-2 receptors isn't physiologic. However, attachment of spike S1 protein of corona virus to the receptor, it has been observed that the receptors are endocytosed into cytoplasm. The receptor virus complex is then transferred to endosomes. There is also a soluble plasma form beside membranous form that can be detected via its extracellular shedding in the plasma and urine. A broad range of proteases has been developed from the SARS-CoV-2, which helps in the cellular entrance of viruses following binding receptors in the body and $S$ protein priming, including cathepsin L, Cathepsin B, trypsin, factor $\mathrm{X}$, elastases and TMPRSS2 [17].

\section{The distribution in human body}

Enterocytes of small membrane, duodenum, proximal tubular cells of the kidneys, glandular cells of the gallbladder, as well as Sertoli cells and Leydig cells of testis [18] are attached to the ACE2 and localize there and helps us to understand their role in SARS-
CoV-2 and ACE-2 receptors in host infected body. Although written above are the sites of ACE2 receptors in human body, it has been discovered through ACE2 mRNA expression via real time PCR that the receptors have been detected in technically all the organs of the body. Although ACE- 2 is regarded as an important regulator of heart function and control of blood pressures [19], possibly operating as a natural compartment of ACE-1[20] the physiological role of ACE-2 has not been clarified in most tissues. ACE-2, which has recently been found as the SARS-coV-2 functional receptor [21], has been found to encourage airway entrance of viruses due to abundance of ACE2 receptors in the respiratory epithelium and in the arterial smooth muscles.

\section{The relationship between ACE2 receptors and organic vulnerability to SARS-CoV-2}

It has now been deduced that the major entry point of the virus is through airways and that the abundance of ACE-2 receptors in the respiratory epithelium serves as the main gateway of entry for it. Lower respiratory tract illness, producing pulmonary and respiratory discomfort, is primarily caused by SARS-CoV-2 [22]. Recent research using the use of viral isolation, cultivation and in situ hybridization have shown the presence of SARS-CoV-2 in pneumocytes [23]. However, the abundance of the receptors in an organ is not directly proportional to the vulnerability of that organ to the infection. For this purpose, a comparative study is undertaken to prove the point. After making a comparative map, it is highlighted that ileum has the highest ACE-2 expression but is not the most vulnerable organ to SARS-CoV-2, leading to think of other multifactorial causes and risks involved in the infection. The possible theories of mechanisms that's 


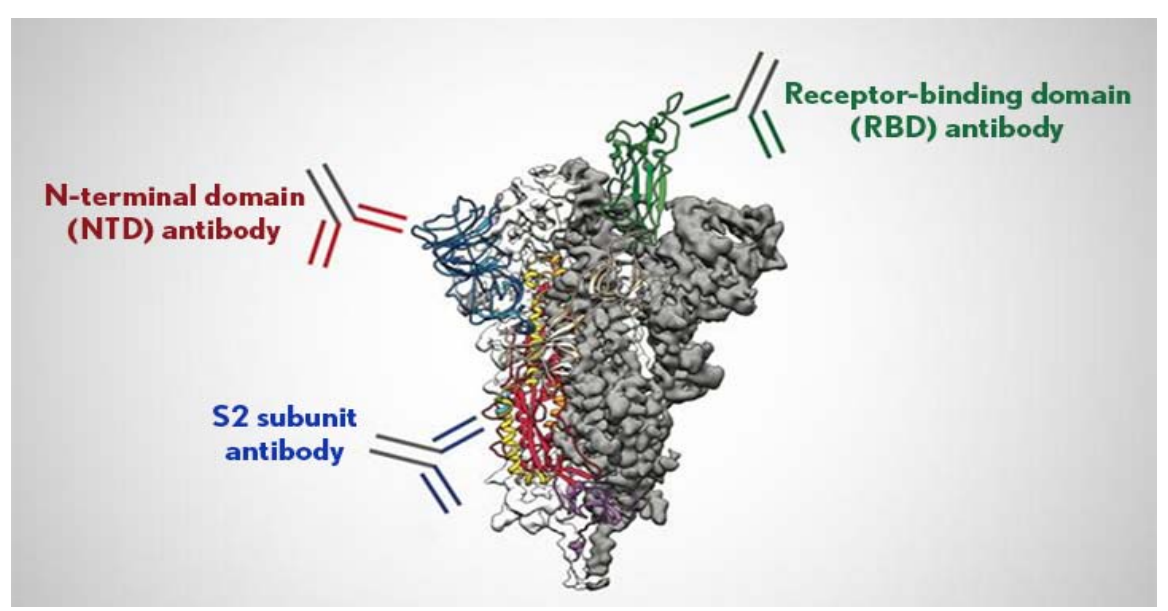

Figure 2: Domain of Spike protein.

https://directorsblog.nih.gov/wp-content/uploads/2021/05/Antibodies-on-spike.jpg

can be thought of as the reason for this inconsistency is: The other receptors might be involved for infection. ACE- 2 might not be the only single factor for the viral infection and it might need assistance of other receptors like angiotensin II receptor i.e., ATR2. The ACE-2 function is regulated by unknown proteins for example $\mathrm{B}^{6} \mathrm{ATI}$ is a neutral amino acid transporter located in the ileum, cause formation of ACE-2 homodimer. This leads to concealing of the ACE-2 ectodomain cleavage-sites, shedding. Consequently, it prevents endocytosis of the receptor, thus, blocking a major pathway in the viral entry. This is how ileum is usually spared in Covid infections. There are some promoter proteins like TMPRSS2 which enhance the bonding/fusion between the activated virus and the host cell.

\section{Proteolytic Transformation and Stimulation of Spike Protein}

Proteolytic transformation of spike proteins is very crucial for the entry and attachment of the virus. In the viral infection, first step virus recognizes the attachment site and binds with it and enter into the cell. In second step, spike protein is split from the cleavage site. It has two subunits named as S1 and S2 then during this process, S2 subunit released [24-26]. In this process, there are many stages included such as the recognition of attachment sites, virus attach on the surface of cell where the attachment sites are present, changing the configuration of spike proteins and last step is to proteolytic activity followed by the spike protein that released the S2 subunit which may lead to fuse the virus and then undergoes endocytosis $[27,28]$. In the initial phase of cellular entry, virus is binding to the cell surface receptors of the spike protein, RBD (Receptor- Binding Domain) of S1 mediated to the ACE-2. The RBD of S1 consists of 200 amino acid domains. This S1 domain give the site for the S2 domain $[29,30]$. The binding of the RBD to the specific domain of ACE-2 named as carboxypeptidase domain. Due to this binding, it moves in a particular orientation such as hinge position between two forms (up and down) and its representation or hide the residues that is linked to the ACE2 receptors [31]. There is the receptor binding motif which is also present within the RBD. This binding motif is necessary for the primary connection with peptidase domain of the ACE-2 [32,33]. The RBM spike protein have conserved sequence approximate the
$50 \%$ of complete sequence between SARS-S and SARS-CoV2-S. The $50 \%$ conserved sequence of RBM of spike protein that is lack from the SARS-related-CoV that do not use the ACE-2. The second phase of this process in which the cleavage of spike protein occurred. During this phase, there are two following step which involved into cleavage, such as priming cleavage and other is activation cleavage. These phases occurred at the cleavage site between the S1 and S2 domains and it is also present into the $\mathrm{S} 2$ region. Most spike proteins in $\mathrm{CoV}$ are followed the cleavage between the assembly of S1 and S2. Multibasic Arginine residues are present between the S1 and S2 cleavage sites. So, the SARS-CoV employs the ACE-2 receptor, SARS-CoV2 contains a unique furin cleavage site (Arg-Arg-Ala- Arg) between the S1 and S2 domains (residues 682-685), that are not present in it and which could explain some of the biochemical changes between SARS-CoV. The removal of this motif has an impact on cellular entrance. Due to the widespread cellular expression of furin proteases, notably in the respiratory tract, the furin cleavage site increases SARS-adaptability CoV-2's for cleavage by cellular proteases, as well as its potential tropism and transmissibility. The virions which are newly synthesized can be released in order to combine with other cells and infect them. This is called preactivated form and they in this process never come in contact with ACE-2 receptor [34]. The process of activation results in $\mathrm{S} 2$ region cleavage that helps in activation of protein for membrane fusion, which is a cell serine transmembrane protease inhibition 2, which in result use camostat mesylate as partial blocker. We all know corona virus infection have a link with TMPRSS2 but this is also a requirement for another influenza infection [35]. Cathepsin B and $\mathrm{L}$ are cellular proteases used to activate the $\mathrm{S}$ protein (endosomal cysteine proteases) of novel virus in this way they can help to decrease and stop the entry of virus in host cell. Sometimes priming proteolysis [36] occurs a process in which many proteases like furin, elastase and factor X also become capable for the start of viral entry and all cellular process but in corona virus the entry in host can occur without any of protease independent mechanisms and receptor dependent. PIKfyve is an enzyme, which controls all early and late endosomal maturations by producing P1, P2 [37]. We can stop viral entry by doing its inhibition [38]. 


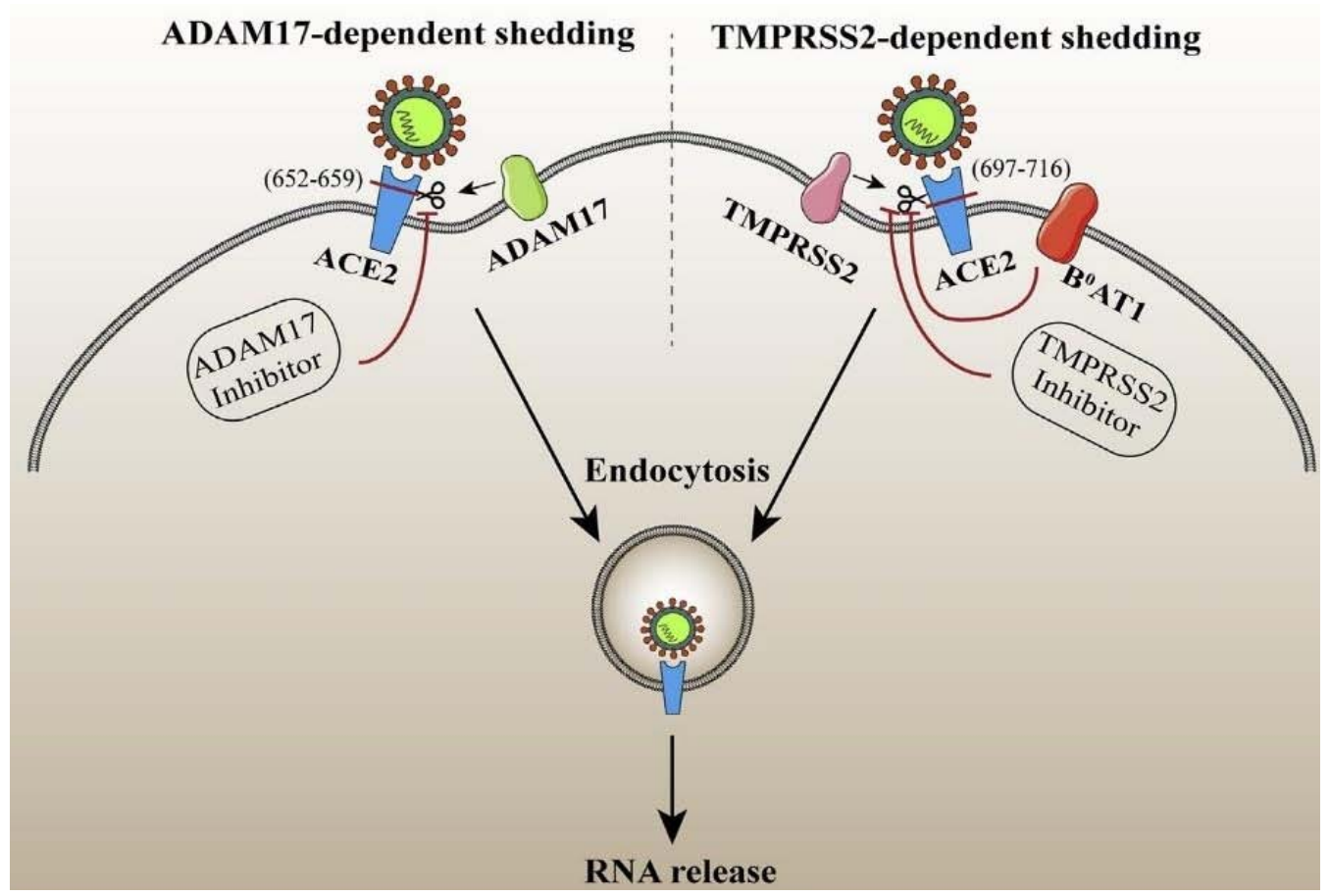

Figure 3: The image shows the residues of arginine and lysine in the ACE-2 receptor from 652 to 659 amino acid cleavage by ADAM17 but in TMPRSS2 the residues can be seen on amino acid from 697 to 716 . B ${ }^{\circ}$ AT1 can also in some cases inhibit YMPRSS2- dependent ACE-2 shedding and act as a neutral amino acid transporter.

\section{Evolution of Corona Virus Spike Protein}

In the evolution of SARS-CoV-2 the S1 domains structure play an important role. Beta-coronavirus S1-NTDs specifies a host origin of coronavirus that is S1-CTDs which is yet less clear in its role. The similarity in structural topologies of $\beta$-sandwich folds is shown by host gelatins and alpha coronavirus S1CTD's. In the diagram below we can see relatively less similarity between S1NTD's and host gelatins. The folds $\beta$-sandwich are common and stable and the convergent evolution of protein stability as the evolutionary drive may produce two $\beta$-sandwich folds. Nevertheless, two sandwich folds with related structural topologies can indicate a common origin if the same order is connected with a substantial number of their $\beta$-strands. S1-CTD and host galectins can therefore be evolutionarily linked. One possible scenario is that S1-NTD is produced through gene capture by the gene duplication of S1-NTD; S1-CTD is produced. As evidenced by the various tertiary structures between alpha and beta coronaviruses S1-CTD, it would appear that S1-CTDs develop at an accelerated pace. Thus, host immune surveillance is under select severe pressure for S1-CTDs to escape. The resulting rapid development of S1-CTDs can, except for the limited information from their structural topology, constantly eliminate their evolutionary traces. The two-domain structure of S1 provides the possibility that coronaviruses have two receptor binding domains, whether or not S1-CTDs originate from host galectins. The S1-NTD uses sugar as a fallback receiver receptor and is more structurally and functionally preserved, while the S1CTD more aggressively evolves and exploits new protein receptors. The structural and functional similarities between $\mathrm{S} 2$ and other fusion proteins from the viral membrane of class I are profound. The prefusion and post-fusion conformations contain these proteins. They can, in a number of similar ways, trigger their pre-fusion structures, undergo similar conformational rearrangement, and transfer to very similar structures with exposed fusion peptides. Although the fact that the same membrane fusion mechanism evolved independently in these viruses cannot be excluded completely, the complexity of this mechanism indicates that viral membrane fusion proteins of class I probably share a common evolutionary ancestor.

\section{Mechanism of ACE2-Mediated Virus Entering Host Cell}

TMPRSS2 gene is located on chromosome 21 encoding enzyme transmembrane protease 2 or serine 2 . It is mainly located on prostate epithelium but has also been found on respiratory tract recently. The most important role in the proliferation and pathogenesis of corona virus is due to TMPRSS2. It was seen that the expression of TMPRSS2 is more in prostate epithelium as compared to any other tissue despite the fact that it was seen in human gut [39]. On the contrary to the usual process of endocytosis-mediated entry of SARS-CoV-2 virus via ACE receptors, during the TMPRSS2 mediated entering, ACE-2 seems to stabilize or fix the virus in the cell surface, thus, enhancing the fusion between the cell and virus. This fusion pathway is also significant in similar viral members i.e., SARS-CoV-2 and MERSCoV-2. Ectodomain can be released from the membrane due to proteolytic shedding of transmembrane protein, which is ACE-2, and a fragment remain bound to the membrane. For the distribution and normal function of proteins proteolytic shedding process is vital. It helps to terminate the full-length membrane protein and interact with its function as well as it activates the membrane protein by releasing ectodomain and in this way it helps and contribute in membrane protein endocytosis $[40,41]$. Shedding can also result in 
faster entry of corona virus. ACE-2 shedding process can increase the viral activity by promoting virus uptake to the targeted cells. ADAM17 is a disintegrin and metalloproteinase it has a great part in regulation and shedding of ACE-2 [42]. For this process, arginine and lysine residues are used within the ACE-2 amino acid 652 till 659 [43]. Different from ADAM17 there is type II transmembrane serine proteases TMPPSS2 which also helps in attachment of SARSCoV-2 spike protein entry with help of ACE-2 receptors. Another factor noticed for the regulation of ACE-2 mediated virus entering the human body is convertase furin, which is a proprotein and preactivates SARS-CoV-2 $\mathrm{S}$ protein and increases the chance of entry of the virus to host body especially during viral packaging. The Figure 1 is showing two modes explained above of ACE- 2 shedding. The shedding induces the process of viral entry to host cells by binding SARS-CoV-2 proteins and these two mechanisms are involved as per research till now.

\section{Conclusion}

Many initial researches that are based on animal's experiments give information about down regulation of ACE-2 expression which is particularly due to corona virus and Spike protein [44]. The counter regulator RAS is ACE-2 and it is its function. The amount of ACE2-Ang (1e7)-Mas R axis decrease with the decrease in ACE-2 expression. This was noticed more after the Corona virus pandemics. After sometime of Corona virus outbreak it was confirmed that Ang II which is found in plasma of virus infected patient higher than those who were tested negative [45]. The physiological and pathophysiological role of this receptor is massive especially for entry in the host cell. High amount of this receptor affects the lungs the most but it attacks many vital organs in human body. Many organs' failure can occur in the patients suspected positive for the virus due to imbalance of RAS and angiotensin enzyme. The S proteins of SARS$\mathrm{CoV}-2$ are amazing molecules like all other class 1 membrane fusion proteins whose function is to enhance the entrance of corona virus in human body to cause infection by binding to ACE-2 receptors. The post trimeric S2 of spike protein us a six- helix bundle containing exposed fusion peptides whereas pre-fusion trimeric spike has three receptor binding $\mathrm{S} 1$ heads along with trimeric membrane fusion S2 stalk. Spike proteins along with the site fir receptor biding engages with ACE-2 receptor during the pathogenesis of novel corona virus. The entry led to priming by TMPRSS2 so fusion of spike proteins can occur with ACE-2 receptors [46]. SARS-CoV-2 virus causes top and decrease respiration illnesses consisting of pneumonia, and in a few cases, results in deadly pulmonary failure. Angiotensin changing enzyme-2, the receptor for mobile access of SARS-CoV-2 virus, has been proven to defend towards intense acute lung failure. Here, we offer proof that SARS-CoV-2 spike protein S1 decreased the mRNA expression of ACE-2 and sort I interferons in number one cells of lung Bronchoalveolar Lavage (BAL) from naïve rhesus macaques. The expression stages of ACE- 2 and sort I interferons have been additionally determined to be correlated with every other, steady with the current locating that ACE-2 is an interferon-inducible gene. Furthermore, induction of ACE-2 and sort I interferons with the aid of using poly I: C, an interferon inducer, turned into suppressed with the aid of using S1 protein in number one cells of BAL. These observations recommend that the downregulation of ACE-2 and sort I interferons triggered with the aid of using S1 protein may also at once make contributions to SARS-CoV-2-related lung illnesses.

\section{References}

1. Yin Y, Wunderink RG. MERS, SARS and other coronaviruses as causes of pneumonia. Respirology. 2018; 23: 130-137.

2. Brian DA, Baric RS. Coronavirus genome structure and replication. Curr Top Microbiol Immunol. 2005; 287: 1-30.

3. Zhu N, Zhang D, Wang W, Li X, Yang B, Song J, et al. A Novel Coronavirus from Patients with Pneumonia in China, 2019. N Engl J Med. 2020; 382: 727-733.

4. Bourgonje AR, Abdulle AE, Timens W, Hillebrands J-L, Navis GJ, Gordijn $\mathrm{SJ}$, et al. Angiotensin converting enzyme 2 (ACE2), SARS-CoV-2 and the pathophysiology of coronavirus disease 2019 (COVID-19). J Pathol. 2020; 251: $228-248$.

5. Li F. Structure, function, and evolution of coronavirus spike proteins. Annu Rev Virol. 2016; 3: 237-261

6. Guo YR, Cao QD, Hong ZS, Tan YY, Chen SD, Jin $\mathrm{HJ}$, et al. The origin, transmission and clinical therapies on coronavirus disease 2019 (COVID-19) outbreak - an update on the status. Mil Med Res. 2020; 7: 11.

7. Walls AC, Park YJ, Tortorici MA, Wall A, McGuire AT, Veesler D. Structure, function, and antigenicity of the SARS-CoV-2 spike glycoprotein. Cell. 2020; 181: 281-292.e6

8. Wrapp D, Wang N, Corbett KS, Goldsmith JA, Hsieh CL, Abiona O, et al Cryo-EM structure of the 2019-nCoV spike in the prefusion conformation. Science. 2020; 367: 1260-1263.

9. Li F. Receptor recognition and cross-species infections of SARS coronavirus Antiviral Res. 2013; 100: 246-254.

10. Wang N, Shi X, Jiang L, Zhang S, Wang D, Tong P, et al. Structure of MERSCoV spike receptor-binding domain complexed with human receptor DPP4. Cell Res. 2013; 23: 986-993.

11. Wang K, Chen W, Zhou Y-S, Lian J-Q, Zhang Z, Du P, et al. SARS-CoV-2 invades host cells via a novel route: CD147-spike protein. BioRxiv. 2020.

12. Yi CE, Ba L, Zhang L, Ho DD, Chen Z. Single amino acid substitutions in the severe acute respiratory syndrome coronavirus spike glycoprotein determine viral entry and immunogenicity of a major neutralizing domain. J Virol. 2005; 79: $11638-11646$

13. Donoghue M, Hsieh F, Baronas E, Godbout K, Gosselin M, Stagliano N, et al. A novel angiotensin- converting enzyme-related carboxypeptidase (ACE2) converts angiotensin I to angiotensin 1-9. Circ. Res. 2000; 87: E1-E9.

14. Crackower MA, Sarao R, Oudit GY, Yagil C, Kozieradzki I, Scanga SE, et al. Angiotensin-converting enzyme 2 is an essential regulator of heart function. Nature. 2002; 417: 822-828

15. Lambert DW, Yarski M, Warner FJ, Thornhill P, Parkin ET, Smith Al, et al. ("Tumor necrosis factor-alpha convertase (ADAM17) mediates regulated ectodomain shedding of the severe-acute respiratory syndrome-coronavirus (SARS-CoV) receptor, angiotensin-converting enzyme-2 (ACE2)". The Journal of Biological Chemistry. 2005; 280: 30113-30119.

16. Patel VB, Clarke N, Wang Z, Fan D, Parajuli N, Basu R, et al. "Angiotensin II induced proteolytic cleavage of myocardial ACE2 is mediated by TACE/ ADAM-17: a positive feedback mechanism in the RAS". Journal of Molecular and Cellular Cardiology. 2014; 66: 167-176.

17. Millet JK, Whittaker GR. Host cell proteases: critical determinants of coronavirus tropism and pathogenesis. Virus Res. 2015; 202: 120-134.

18. Hikmet Feria, Méar Loren, Edvinsson Åsa, Micke Patrick, Uhlén Mathias, Lindskog Cecilia. "The protein expression profile of ACE2 in human tissues". Molecular Systems Biology. 2020; 16: e9610.

19. Crackower MA, Sarao R, Oudit GY, et al. Angiotensin-converting enzyme 2 is an essential regulator of heart function. Nature. 2002; 417: 822-828.

20. Yagil Y, Yagil C. Hypothesis: ACE2 modulates blood pressure in the mammalian organism. Hypertension. 2003; 41: 871-873. 
21. Li W, Moore MJ, Vasilieva N, et al. Angiotensin-converting enzyme 2 is a functional receptor for the SARS coronavirus. Nature. 2003; 426: 450-454.

22. Ding $\mathrm{Y}$, Wang $\mathrm{H}$, Shen $\mathrm{H}$, et al. The clinical pathology of severe acute respiratory syndrome (SARS): a report from China. J Pathol. 2003; 200: 282 289.

23. To KF, Tong JHM, Chan PKS, et al. Tissue and cellular tropism of the coronavirus associated with severe acute respiratory syndrome: an in-situ hybridization study of fatal cases. J Pathol. 2004; 202: 157-163.

24. Wang Q, Zhang Y, Wu L, et al. Structural and functional basis of SARSCoV-2 entry by using human ACE2. Cell. 2020; 181: 894-904.e9.

25. Shang J, Ye G, Shi K, et al. Structural basis of receptor recognition by SARS CoV-2. Nature. 2020; 581: 221-224.

26. Yan R, Zhang Y, Li Y, et al. Structural basis for the recognition of SARS CoV-2 by full- length human ACE2. Science. 2020; 367: 1444-1448.

27. Belouzard S, Chu VC, Whittaker GR. Activation of the SARS coronavirus spike protein via sequential proteolytic cleavage at two distinct sites. Proc Natl Acad Sci U S A. 2009; 106: 5871-5876.

28. Hoffmann M, Kleine-Weber H, Schroeder S, et al. SARS-CoV-2 cell entry depends on ACE2 and TMPRSS 2 and is blocked by a clinically proven protease inhibitor. Cell. 2020; 181: 271-280.

29. Wang Q, Zhang $Y$, Wu L, et al. Structural and functional basis of SARS CoV-2 entry by using human ACE2. Cell. 2020; 181: 894-904.e9.

30. Wrapp D, Wang N, Corbett KS, et al. Cryo-Em structure of the 2019-nCoV spike in the prefusion conformation. Science. 2020; 367: 1260-1263.

31. Li F, et al. Structure of SARS coronavirus spike receptor-binding domain complexed with receptor. Science. 2005; 309: 1864-1868.

32. Li W, Zhang C, Sui J, et al. Receptor and viral determinants of SARS coronavirus adaptation to human ACE2. Embo J. 2005; 24: 1634-1643.

33. Walls AC, Park Y-J, Tortorici MA, et al. Structure, function, and antigenicity of the SARS- CoV-2 spike glycoprotein. Cell. 2020; 181: 281-292.

34. Hoffmann M, Kleine-Weber $\mathrm{H}$, Schroeder S, et al. SARS-CoV-2 cell entry depends on ACE2 and TMPRSS2 and is blocked by a clinically proven protease inhibitor. Cell. 2020; 181: 271-280.
35. Millet JK, Whittaker GR. Host cell entry of Middle East respiratory syndrome coronavirus after two-step, furin-mediated activation of the spike protein. Proc Natl Acad Sci U S A. 2014; 111: 15214-15219.

36. Millet JK, Whittaker GR. Host cell proteases: critical determinants of coronavirus tropism and pathogenesis. Virus Res. 2015; 202: 120-134

37. Ou X, Liu $Y$, Lei $X$, et al. Characterization of spike glycoprotein of SARSCoV-2 on virus entry and its immune cross-reactivity with SARS-CoV. Nat Commun. 2020; 11: 1620

38. COVID-19 (SARS-CoV-2) Spike RBD Recombinant Protein

39. Xu H, Zhong L, Deng J. et al. High expression of ACE2 receptor of 2019nCoV on the epithelial cells of oral mucosa. Int. J. Oral Sci. 2020; 12: 8.

40. Lichtenthaler SF, Lemberg MK, Fluhrer R. Proteolytic ectodomain shedding of membrane proteins in mammals-hardware, concepts, and recent developments. EMBO J. 2018; 37: e99456.

41. Clark P. Protease-mediated ectodomain shedding. Thorax. 2014; 69: 682e4.

42. Xu J, Mukerjee S, Silva-Alves CR, Carvalho-Galvao A, Cruz JC, Balarini CM et al. A disintegrin and metalloprotease 17 in the cardiovascular and central nervous systems. Front Physiol. 2016; 7: 469.

43. Heurich A, Hofmann-Winkler H, Gierer S, Liepold T, Jahn O, Pohlmann S. TMPRSS2 and ADAM17 cleave ACE2 differentially and only proteolysis by TMPRSS2 augments entry driven by the severe acute respiratory syndrome coronavirus spike protein. J Virol. 2014; 88: 1293-1307.

44. Kuba K, Imai Y, Rao S, Gao H, Guo F, Guan B, et al. A crucial role of angiotensin converting enzyme 2 (ACE2) in SARS coronavirus induced lung injury. Nat Med. 2005; 11: 875- 879.

45. Liu Y, Yang Y, Zhang C, Huang F, Wang F, Yuan J, et al. Clinical and biochemical indexes from 2019-nCoV infected patients linked to viral loads and lung injury. Sci China Life Sci. 2020; 63: 364-374.

46. Oudit GY, Kassiri Z, Jiang C, Liu PP, Poutanen SM, Penninger JM, et al. SARS-coronavirus modulation of myocardial ACE2 expression and inflammation in patients with SARS. Eur J Clin Invest. 2009; 39: 618-625. 